\title{
Minimally Invasive Sacroiliac Joint Fusion with Triangular Titanium Implants: Cost-Utility Analysis from NHS Perspective
}

\author{
Deirdre B. Blissett ${ }^{1}$ (i) $\cdot$ Rob S. Blissett ${ }^{1} \cdot$ Matthew P. Newton Ede ${ }^{2,3} \cdot$ Philip M. Stott $^{4} \cdot$ Daniel J. Cher $^{5}$. \\ W. Carlton Reckling ${ }^{6}$
}

Accepted: 15 October 2020 / Published online: 9 November 2020

(c) The Author(s) 2020

\begin{abstract}
Objective The aim was to identify the cost-effectiveness of minimally invasive sacroiliac joint fusion (MI SIJF) surgery with titanium triangular implants for patients with sacroiliac joint (SIJ) pain who have failed conservative management, compared to non-surgical management (NSM) from a National Health Service (NHS) England perspective.

Methods Over a time horizon of 5 years, a cohort state transition model compared the costs and outcomes of treating patients with MI SIJF to those of traditional NSM treatment pathways. The NSM arm included two treatments: grouped physical therapy and corticosteroid injections (PTSI) or radiofrequency ablation (RFA). Three different strategies were considered: (1) a stepped pathway, (2) patients split between PTSI and RFA, and (3) RFA only. The outcome measure was incremental cost-effectiveness ratio (ICER), reported in 2018 British pounds per quality-adjusted life year (QALY) gained. One-way and probabilistic sensitivity analyses were used to test the robustness of the model results.

Results Patients undergoing MI SIJF accrued total procedure-related and pain-management costs of $£ 8358$, while NSM treatment strategy 1 had total costs of $£ 6880$. The MI SIJF cohort had 2.98 QALYs compared to strategy 1 with 2.30 QALYs. This resulted in an ICER for MI SIJF versus strategy 1 of £2164/QALY gained. Strategy 2 of the NSM arm had lower costs than strategy 1 (£6564) and 2.26 QALYs, and this resulted in an ICER of £2468/QALY gained for MI SIJF. Strategy 3 of the NSM arm had lower costs than strategy 1 ( $£ 6580)$, and this resulted in 2.28 QALYs and an ICER of £2518/QALY gained for MI SIJF. Probabilistic sensitivity analysis shows that at a threshold of $£ 20,000 / Q A L Y$ gained, MI SIJF has a probability of being cost-effective versus NSM strategies of $96 \%, 97 \%$, and $91 \%$ for strategies 1,2, and 3, respectively.

Conclusion MI SIJF appears to be cost-effective over a 5-year time horizon when compared to traditional NSM pathways in an NHS context.
\end{abstract}

Electronic supplementary material The online version of this article (https://doi.org/10.1007/s41669-020-00236-5) contains supplementary material, which is available to authorized users.

Deirdre B. Blissett

deirdre.blissett@medtecheconomics.co.uk

MedTech Economics Ltd., Winchester, UK

2 The Royal Orthopaedic Hospital, Bristol Road South, Birmingham, UK

3 Precision Spine, 81 Harborne Road, Edgbaston, Birmingham, UK

4 Brighton and Sussex University Hospitals NHS Trust, Eastern Road, Brighton, UK

5 Department of Clinical Affairs, SI-BONE, Inc., San Jose, CA, USA

6 Department of Medical Affairs, SI-BONE, Inc., San Jose, CA, USA

\section{Key Points for Decision Makers}

In patients with sacroiliac joint (SIJ) pain who have failed conservative management, patients treated with minimally invasive sacroiliac joint fusion (MI SIJF) are estimated to accrue total procedure-related and pain-management costs of $£ 8358$ over a 5-year period compared to non-surgical management (NSM) costs of $£ 6880$ over the same period.

Treatment with MI SIJF results in quality-adjusted life year (QALY) gains of 2.98 QALYs compared to 2.30 QALYs with NSM over the same period.

MI SIJF appears to be cost-effective over a 5-year time horizon when compared to traditional NSM pathways in a National Health Service (NHS) context. 


\section{Introduction}

Lower back pain affects up to a third of the UK adult population [1], and sacroiliac joint (SIJ) pain is estimated to affect between 15 and 30\% of sufferers of chronic lower back pain [2]. Amongst those with continued or new onset lower back pain after lumbar fusion, the prevalence of SIJ pain is even higher at $32-43 \%[3,4]$. The cost to the UK economy attributable to lower back pain has been estimated at $£ 12.3$ billion in 1998 [5, 6], equivalent to $£ 38.4$ billion in 2018 . Lower back pain is also ranked within the global top ten disease areas with the highest impact on disability-adjusted life years [7].

Nonsurgical management (NSM) for chronic SIJ pain include medical management, physical therapy, intra-articular or peri-articular steroid injections, and radiofrequency ablation (RFA) of sacral nerve root branches. However, the evidence base supporting the efficacy of these interventions is limited [8-12].

Surgical fusion of the SIJ has been carried out since the 1920s. Several single-centre, retrospective case series suggest modest efficacy for open SIJ fusion, combined with a high morbidity rate such that open SIJ fusion is no longer commonly used [13]. There is a large body of compelling clinical evidence demonstrating minimally invasive SIJ fusion (MI SIJF), using titanium triangular implants (TTIs) (iFuse Implant System, SI-BONE, Santa Clara, CA, USA) delivers benefit to patients with confirmed SIJ pain. The National Institute for Health and Care Excellence (NICE) interventional procedure guidance [14] recommends SIJ fusion using a lateral transarticular approach. There are several available devices for MI SIJ fusion that are placed laterally. However, the majority of the clinical evidence which supports the safety and effectiveness of the procedure, including multiple randomized controlled clinical trials (RCTs) [15, 16], a large prospective multicentre study [17], multiple comparative cohorts [17-19], and long-term studies, evaluated procedures performed with TTIs. Results from these surgeries indicate that MI SIJ fusion results in a substantial immediate and sustained improvement in pain, disability, and quality of life. The procedure to insert TTIs takes about an hour and is performed under general anaesthetic. Typically, three implants are placed across the SIJ through a small incision $(<6 \mathrm{~cm})$.

In 2018, NICE evaluated MI SIJF for treating chronic severe SIJ pain [22] under the Medical Technology Evaluation Programme (MTEP), concluding that MI SIJF results in pain relief, less disability, and improved quality of life compared with NSM. The results of a cost-minimization analysis suggested that while MI SIJF is initially more expensive, at 8 years and beyond, it becomes cost saving when compared to non-operative management. A positive recommendation under this NICE programme does not oblige National Health Service (NHS) England decision makers to make funding available. Showing evidence of cost-effectiveness over a shorter time horizon will be helpful to facilitate the decisionmaking process, demonstrating that MI SIJF is cost-effective in the short and medium term when the utility benefits are considered.

The objective of this analysis is to incorporate both clinical outcome and cost data in a cost-effectiveness model to determine the short- and medium-term clinical and economic benefits of MI SIJF.

\section{Methods}

\subsection{Model Design}

We constructed a Markov decision model in Microsoft Excel to estimate 5-year costs (2018 British pounds) and quality-adjusted life years (QALYs) from an NHS perspective with 3-month model cycles. Both costs and QALYs were discounted by $3.5 \%$ as per the recommendations in the NICE reference case. The model population was adults with chronic, disabling SIJ pain unrelated to acute trauma or underlying inflammatory disease who have failed conservative management. The population had a mean age of 50 years and a baseline Oswestry Disability Index (ODI) of 56.1 as per the trial populations of the INSITE [15], SIFI [17], and iMIA [16] trials.

The model structure described in the Medical Technology Guidance (MTG) 39 External Assessment Centre (EAC) report [23] was adapted to capture health state utility, and is illustrated in Figs. 1 and 2. A Markov structure was selected as it allows modelling of transitions in health states over fixed periods of time. This is relevant to this decision problem for the following reasons: (1) patients appear to transition from highly symptomatic to much less symptomatic within a month or two post SIJF; (2) patients treated with steroid injections in the physical therapy and corticosteroid injections (PTSI) arm may show some temporary improvement, but often worsen quickly; and (3) patients treated with RFA show somewhat longer periods of response, but then routinely worsen over time.

A 3-month time horizon was applied to allow patients to transition from a health state for responding well to treatment to a health state for recurring pain over a relatively short time period. It is assumed that treatment in any of the interventions occurs in a 3-month period, during which the patient maintains baseline utility scores, but does not incur additional pain-management costs on top of their intervention. This is because patients with a response to treatment with MI SIJF experience symptom relief within 1 month of 
surgery. Similarly, patients' immediate and temporary pain relief is assumed with steroid injections and RFA. After the 3-month treatment period, if a patient moves to a response state, it is assumed that they do not incur further healthcare and pharmacy costs related to SIJ pain management, but if they move to a poor ODI response state, their pain-management costs revert to baseline. Patients with chronic SIJ joint pain were assumed not to experience relief from pain naturally, and patients in a response state after surgery were assumed not to experience any deterioration in condition over time. Mortality was modelled as per the general population, no mortality hazard ratio for SIJ joint pain patients was applied, and there was no difference between patients experiencing a response to treatment and those remaining in pain states, an assumption biasing against effective treatments because disability is associated with higher comorbidity.

In the MI SIJF treatment arm, patients enter the model in the surgical health state. This is a tunnel state, and after one model cycle, patients achieve a response to treatment, they fail to achieve an ODI response, or they require an early surgical revision for implant malposition (which can cause new onset radicular pain). Patients indefinitely remain in these health states unless they require a late revision surgery, after which they may move to 'response' or 'no response'. Late revision surgery is occasionally performed to address late recurrence of pain or disability [24], which is captured in the model.

MI SIJF was compared to NSM, which included three alternative treatment strategies:

1. A stepped pathway where patients are initially treated with a combination of conservative management techniques such as PTSI and/or RFA and may transition from treatment with PTSI to RFA

2. A combination pathway where a proportion of patients are treated with PTSI and are not allowed to transition to RFA and a proportion of patients start treatment with RFA

3. Treatment with RFA only

Fig. 1 Diagram of model structure used to simulate patient history following surgery with MI SIJF. MI SIJF minimally invasive sacroiliac joint fusion
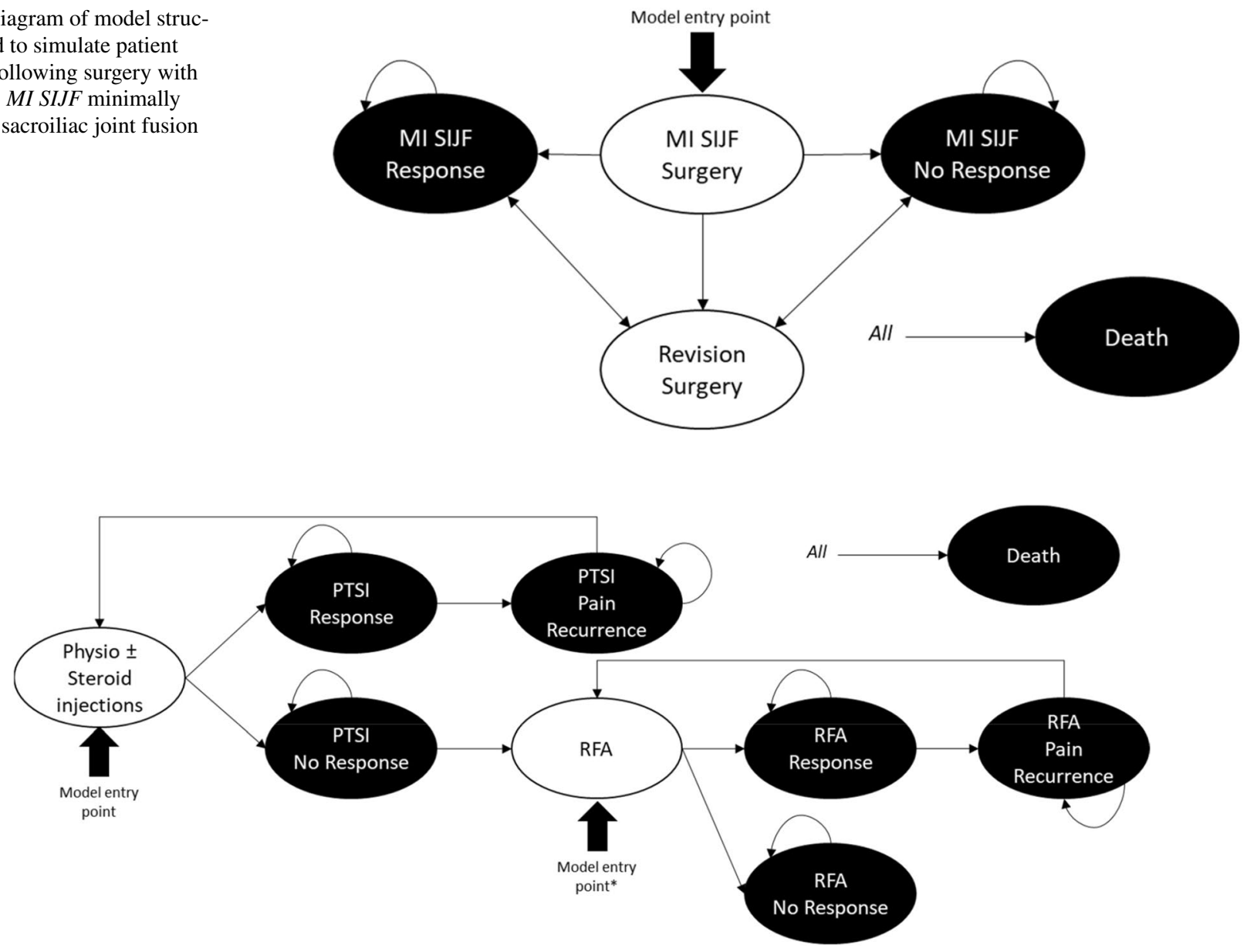

Fig. 2 Diagram of model structure used to simulate patient history through a non-surgical intervention pathway. PTSI physical therapy and corticosteroid injections, RFA radiofrequency ablation. *Model entry point only used for comparator strategies 2 and 3 
Feedback from UK consultants interviewed prior to developing the model (details in the electronic Supplementary material) suggests a stepped pathway is the most common practice in the NHS in England. The latter two strategies were also considered in the model to reflect variation in practice because steroid injections or RFA may not be funded in some localities due to mixed perceptions on their efficacy.

The NSM arm includes nine health states, four for each of the two interventions (PTSI or RFA) plus death. In strategy 1 , all patients start in the PTSI health state, which is assumed to be the standard first-line treatment. They then either achieve an ODI response of $\geq 15$ points or fail to do so. Responders remain in this health state for the duration of the treatment effect, after which they transition to the pain recurrence state and receive a repeat procedure on the next cycle. Where first-line treatment of PTSI fails, patients may stay in the no response health state or progress to RFA as a second-line treatment where the model structure mirrors that for PTSI. It is assumed that there is a $20 \%$ probability of them undergoing RFA in each subsequent model cycle, reflecting feedback provided by the UK consultants interviewed (see the electronic supplementary material). However, in strategies 2 and 3, this probability is assumed to be $0 \%$, to consider localities where RFA is not funded. In strategy 2, 50\% of the patients start in PTSI and 50\% in RFA, and in strategy 3, all patients start in RFA. For both PTSI and RFA, it was assumed that $100 \%$ of patients receiving a positive treatment response to PTSI or RFA would have repeat interventions when the treatment effect wore off. The duration of treatment effect for PTSI required an assumption due to the low quality of evidence; it was assumed, based on available literature, that the median duration of effect was 3 months $[25,26]$. The median duration of effect for RFA was assumed to be 7.9 months [39].

\subsection{Model Parameters}

Details of all cost inputs and sources that are used in the base-case model are reported in Tables 1 and 2 .

\subsubsection{Outcomes}

The minimum clinically important difference (MCID) after SIJ treatment was estimated as a reduction of $\geq 15$ points in a patient's ODI irrespective of baseline [27]. For the purposes of this study and to consider effect at similar time points for MI SIJ and RFA, this reduction in ODI was observed 3 months after the initial intervention.

The effectiveness measures for MI SIJF were sourced from the published literature, focusing primarily on three prospective clinical trials, the most robust published evidence on MI SIJF at the time of publication. Detailed descriptions of the trial protocols are provided elsewhere [28].

Briefly, INSITE was a prospective, 2-year, multicentre RCT conducted at 19 centres in the USA. It included 148 patients with diagnosed SIJ dysfunction unresponsive to at least 6 months of conservative care. Subjects were randomized in a 2:1 fashion to either SIJF or NSM. NSM included anti-inflammatory and opioid pain medications, physical therapy, intra-articular SIJ steroid injections, and RFA, delivered serially as needed to manage pain and disability.

SIFI was a prospective, multicentre, single-arm clinical trial $(n=172)$ conducted at 26 centres in the USA. Study parameters were identical to those of INSITE.

iMIA, a prospective, multicentre RCT ( $n=103$ ), was conducted at nine European centres. Key differences between iMIA and INSITE include iMIA using 1:1 randomization and the NSM arm in iMIA including only physical therapy per European guidelines [29]. There is 'moderate' level evidence for the short-term effectiveness of intra-articular SIJ injections [8]. Because of the paucity of literature, the use of steroid injections was grouped together with physical therapy and response rates for the grouped set of treatments were based on responses observed in the NSM arm in the two MI SIJF RCTs. Although the protocols were different, the data were pooled for convenience. This is likely to be a conservative approach because of the $45.7 \%$ of patients in the INSITE study who underwent RFA. This is likely to have bolstered outcomes for these patients. The current evidence suggests RFA can provide temporary pain relief, but there remains variability in patient selection, nerves targeted, and types of RFA technology and techniques utilized [11, 12]. Effectiveness data for RFA were sourced from an RCT that assessed the efficacy of lateral branch neurotomy for chronic SIJ pain against placebo [30]. There are two recent RCTs that demonstrated minimal effectiveness of SIJ RFA [31, 32].

In England, the preferred outcome measure in economic evaluation is QALYs. The NICE reference case prefers the utility to be measured using the Euro-Qol EQ-5D questionnaire. Although both INSITE and iMIA collected EQ-5D-3L data valued using the UK tariff set at baseline and followup, health utility data following an RFA intervention were not found in the published literature. Therefore, to allow the comparison to RFA, a mapping procedure was used in which EQ-5D-3L data collected from MI SIJF clinical trials (INSITE [15] and iMIA [16]) were linearly regressed against a patient's ODI score. Patient baseline characteristics were incorporated in the model, and only age was found to be a significant additional predictor of EQ-5D-3L value (data illustrated in the electronic Supplementary material). This approach was similar to the indirect method to determine utilities with RFA reported in the NICE cost-effectiveness analysis of RFA [33]. 
Table 1 Clinical effectiveness measures

\begin{tabular}{|c|c|c|c|}
\hline & & Distribution & Source \\
\hline \multicolumn{4}{|l|}{ Response to procedure } \\
\hline MI SIJF & $65.4 \%$ & Beta $(210,111)$ & 3-month response rate. Data pooled from INSITE [15], iMIA [16], and SIFI [17] \\
\hline PTSI & $25.0 \%$ & Beta $(24.25,72.75)$ & 3-month response rate. Data pooled from INSITE [15] and iMIA [16] \\
\hline RFA & $26.0 \%$ & Beta $(9,25)$ & 3-month response rate. Patel et al. [30] \\
\hline \multicolumn{4}{|c|}{ ODI change by health state ${ }^{1}$} \\
\hline Response: MI SIJF & -33.3 & Normal $(-33.3,12.5)$ & Data pooled from INSITE [15] and iMIA [16]. No treatment effect observed \\
\hline No response: MI SIJF & -1.9 & Normal $(-1.9,9.2)$ & Data pooled from INSITE [15] and iMIA [16]. No treatment effect observed \\
\hline Response: PTSI & -33.3 & Normal $(-33.3,12.5)$ & Data pooled from INSITE [15] and iMIA [16]. No treatment effect observed \\
\hline No response PTSI & -1.9 & Normal $(-1.9,9.2)$ & Data pooled from INSITE [15] and iMIA [16]. No treatment effect observed \\
\hline Response: RFA & -33.0 & Normal $(-33.0,9.22)$ & {$[30]$} \\
\hline No response: RFA & -1.64 & Normal $(-1.64,11.03)$ & {$[30]$} \\
\hline \multicolumn{4}{|c|}{ Duration of treatment effect (months) } \\
\hline PTSI & 3 & Log normal $(1.10,0.35)^{2}$ & {$[25,26]$} \\
\hline RFA & 7.9 & Log normal $(2.07,0.10)$ & [39] \\
\hline \multicolumn{4}{|l|}{ Risk of revision surgery } \\
\hline Risk of early revision ${ }^{3}$ & $0.81 \%$ & Beta $(10,490,85)$ & {$[24,40]$} \\
\hline Risk of late revision ${ }^{4}$ & $0.17 \%$ & $(10,490,18)$ & {$[24,40]$} \\
\hline
\end{tabular}

$C I$ confidence interval, MI SIJF minimally invasive sacroiliac joint fusion, ODI Oswestry Disability Index, PTSI physiotherapy + corticosteroid injection, $R F A$ radiofrequency ablation

${ }^{1}$ Negative values refer to improvement in scores

${ }^{2}$ Assumed a $95 \% \mathrm{CI}$ of $\pm 33 \%$

${ }^{3}$ Cumulative risk of symptomatic malposition at 12 months. Assumed to occur in subsequent model cycle

${ }^{4}$ Cumalative risk of revision surgery for other reasons at 12 months. Converted to 3-month probability

$\mathrm{EQ}-5 \mathrm{D}=0.92-0.009 \times \mathrm{ODI}+0.0011 \times$ Age

\subsubsection{Costs}

The total hospital length of stay, procedure times, surgical training hours, and follow-up consultations for MI SIJF were derived from the clinical literature and were used in the NICE MTG39 EAC analysis [22]. The number of consumables required for each MI SIJF surgery was estimated in consultation with clinical experts (further details reported in the electronic supplementary material). The cost of surgery-related adverse events was not considered separately in the model because adverse events following MI SIJF surgery are very low and mainly related to wound problems treated in hospital; therefore, the cost of treatment was already captured in the hospital stay cost.

All the resources were costed using routine NHS costs and prices. Where costs were available in the NHS reference cost schedule, these were taken from the latest $2017 / 2018$ costs. Where costs were taken from elsewhere, they were inflated to costs in 2018 from the year of publication using the UK consumer price index (CPI) health indices available from the Office of National Statistics.

\subsection{Analytical Methods}

Transition probabilities were derived using values from published literature; the values used in the model are presented in Table 3. Where probabilities required converting from one time period to another, the probability was first converted to a 3-month rate and then translated into the corresponding transition probability [34].

To test the impact of the 5-year time horizon, results are also reported over a lifetime as a sensitivity analysis. Benefits of MI SIJF have been shown to be durable up to 5 years $[20,21]$. Longer term follow-up data are not available, but it is reasonable to expect the effect of surgical intervention to persist. Therefore, reporting results at 5 years is a conservative approach, which is aimed at reducing the uncertainty over an NSM pathway over the long term.

Deterministic sensitivity analysis (DSA) was performed on all model inputs, varying each input individual to $20 \%$ of the base-case value. Although this approach does not test all inputs to the maximum range of uncertainty by varying within the 95\% confidence intervals, this approach was used to identify the model parameters particularly important to model outcomes. 
Table 2 Cost inputs and sources

\begin{tabular}{|c|c|c|c|c|c|c|c|c|c|}
\hline & Unit cost & Distribution & Source & $\begin{array}{l}\text { Any use } \\
(\%)\end{array}$ & Distribution & Source & Mean use & Distribution & Source \\
\hline \multicolumn{10}{|l|}{ MI SIJF } \\
\hline $\begin{array}{l}\text { Procedure } \\
\text { (min) }\end{array}$ & $£ 20.00$ & Gamma $(14,1)$ & [41] & 100 & - & - & 59 & $\begin{array}{l}\text { Log normal } \\
\quad(4.08,0.07)\end{array}$ & {$[42]$} \\
\hline $\begin{array}{l}\text { Length of stay } \\
\text { (days) }\end{array}$ & $£ 366.19$ & Gamma $(66,6)$ & $\mathrm{HN} 13 \mathrm{~A}-\mathrm{F}^{\mathrm{a}}$ & 100 & - & - & 0.80 & $\begin{array}{l}\text { Log normal } \\
\quad(-0.22,0.85)\end{array}$ & [22] \\
\hline Training (h) & $£ 108.00$ & Gamma $(44,2)$ & [43] & 1.11 & $\begin{array}{l}\text { Log normal } \\
\quad(0.13,1.39)\end{array}$ & Assumption & 4 & $\begin{array}{l}\text { Log normal } \\
\quad(1.39,0.13)\end{array}$ & {$[42]$} \\
\hline $\begin{array}{l}\text { Surgical } \\
\text { implants (-) }\end{array}$ & $£ 1155.00$ & - & b & 100 & - & - & 3 & - & $\mathrm{b}$ \\
\hline $\begin{array}{l}\text { Surgical acces- } \\
\text { sories }(-)\end{array}$ & $£ 275.00$ & - & $\mathrm{b}$ & 100 & & & 1 & - & b \\
\hline $\begin{array}{c}\text { Steinmann } \\
\text { pins (-) }\end{array}$ & $£ 47.00$ & - & $\mathrm{b}$ & 100 & & & 3 & - & b \\
\hline $\begin{array}{l}\text { Exchange pin } \\
(-)\end{array}$ & $£ 47.00$ & - & $\mathrm{b}$ & 100 & & & 1 & - & $\mathrm{b}$ \\
\hline Drills (-) & $£ 131.00$ & - & $\mathrm{b}$ & 100 & & & 1 & - & b \\
\hline $\begin{array}{l}\text { Pre-surgery } \\
\text { consult } \\
\text { (visits) }\end{array}$ & $£ 146.00$ & $\operatorname{Gamma}(96,2)$ & $\mathrm{WF} 1 \mathrm{~B}^{\mathrm{a}}$ & 100 & - & - & 1 & - & [42] \\
\hline $\begin{array}{l}\text { Post-surgery } \\
\text { follow-up } \\
\text { (visits) }\end{array}$ & $£ 119.00$ & Gamma $(96,1)$ & WF001A ${ }^{a}$ & 100 & - & - & 4 & $\begin{array}{l}\text { Log normal } \\
\quad(1.39,0.13)\end{array}$ & [42] \\
\hline \multicolumn{10}{|c|}{ Corticosteroid injections } \\
\hline Procedure (-) & $£ 397.00$ & Gamma $(96,4)$ & $\mathrm{HC} 29 \mathrm{~B}^{\mathrm{a}}$ & 73.9 & Beta $(34,12)$ & {$[44]$} & 1 & - & - \\
\hline $\begin{array}{l}\text { Pre-procedure } \\
\text { consult } \\
\text { (visit) }\end{array}$ & $£ 193.00$ & Gamma $(96,2)$ & $\mathrm{WF} 01 \mathrm{~B}^{\mathrm{a}}$ & 73.9 & Beta $(34,12)$ & [44] & 1 & - & - \\
\hline \multicolumn{10}{|c|}{ Radiofrequency ablation } \\
\hline Procedure (-) & $£ 776.14$ & Gamma $(96,8)$ & $\begin{array}{l}\mathrm{AB} 15 \mathrm{Z}, \\
\text { AB16Z }\end{array}$ & $100-$ & - & & 1 & - & - \\
\hline $\begin{array}{l}\text { Pre-procedure } \\
\text { consult } \\
\text { (visit) }\end{array}$ & $£ 193.00$ & Gamma $(96,2)$ & $\mathrm{WF} 1 \mathrm{~B}^{\mathrm{a}}$ & 73.9 & Beta $(34,12)$ & {$[44]$} & 1 & - & - \\
\hline \multicolumn{10}{|l|}{ Physiotherapy } \\
\hline $\begin{array}{l}\text { Initial physi- } \\
\text { otherapy ses- } \\
\text { sion (visit) }\end{array}$ & $£ 61.00$ & $\begin{array}{l}\text { Gamma (96, } \\
0.6)\end{array}$ & $\mathrm{WF} 01 \mathrm{~B}^{\mathrm{a}}$ & 97.8 & Beta $(45,1)$ & [44] & 1 & Not varied & {$[45]$} \\
\hline $\begin{array}{l}\text { Follow-up } \\
\text { physiother- } \\
\text { apy session } \\
\text { (visit) }\end{array}$ & $£ 52.00$ & $\begin{array}{l}\text { Gamma }(96, \\
0.5)\end{array}$ & $\mathrm{WF} 1 \mathrm{~A}^{\mathrm{a}}$ & 97.8 & Beta $(45,1)$ & {$[44]$} & 5 & $\begin{array}{l}\text { Log normal } \\
\quad(1.61,0.22)\end{array}$ & {$[45]$} \\
\hline \multicolumn{10}{|c|}{ Resource use in no response health state } \\
\hline $\begin{array}{l}\text { Medica- } \\
\text { tion (£ per } \\
\text { month) }\end{array}$ & $£ 30.80$ & $\begin{array}{l}\text { Gamma }(5.3 \text {, } \\
5.8)\end{array}$ & {$[45]$} & 100 & - & - & 1 & - & - \\
\hline $\begin{array}{l}\text { Pain-man- } \\
\text { agement } \\
\text { resource } \\
\text { cost (£ per } \\
\text { month) }\end{array}$ & $£ 83.92$ & $\begin{array}{l}\text { Gamma (8.8, } \\
9.5)\end{array}$ & [45] & 100 & - & - & 1 & - & - \\
\hline
\end{tabular}

Min minutes, MI SIJF minimally invasive sacroiliac joint fusion, NHS National Health Service

${ }^{a}$ NHS reference costs 2017/2018

${ }^{\mathrm{b}}$ The consumable costs are taken from SI-BONE UK list prices, and the resources required to perform each surgery were informed via consultation with clinical experts 
Table 3 Transition probabilities used in the model

\begin{tabular}{llll}
\hline Starting state & Ending state & Value & Source \\
\hline iFuse model & & & \\
MI SIJF* & MI SIJF response & 0.6487 & a \\
& MI SIJF no response & 0.3432 & a \\
& Revision surgery & 0.0081 & b \\
MI SIJF response & Revision surgery & 0.0017 & c \\
MI SIJF no response & Revision surgery & 0.0017 & c \\
Revision surgery & MI SIJF response & 0.6540 & d \\
& MI SIJF no response & 0.3460 & d \\
NSM model & & & \\
PTSI* & PTSI response & 0.2500 & e \\
& PTSI no response & 0.7500 & e \\
PTSI response & PTSI pain recurrence & 0.5000 & f \\
PTSI no response & RFA & 0.2000 & $\mathrm{~g}$ \\
PTSI pain recurrence & PTSI & 1.0000 & h \\
RFA* & RFA response & 0.2600 & i \\
& RFA no response & 0.7400 & i \\
RFA response & RFA pain recurrence & 0.2314 & j \\
RFA pain recurrence & RFA & 1.0000 & h \\
\hline
\end{tabular}

MI SIJF minimally invasive sacroiliac joint fusion, PTSI physiotherapy + corticosteroid injection, $R F A$ radiofrequency ablation

*These intervention states are both potential entry points to the model and tunnel states

${ }^{\text {a }}$ Probability of response to treatment pooled from INSITE/iMIA/SIFI [1-3] adjusted for proportion of early revisions [4]

${ }^{\mathrm{b}}$ Probability of early revision [4]

${ }^{\mathrm{c}}$ Probability of late revision [4] converted from 24-month to 3-month probability using methods in [5]

${ }^{\mathrm{d}}$ Probability of response to treatment pooled from INSITE/iMIA/ [1-3]

${ }^{\text {e}}$ Probability of response to treatment pooled from INSITE/iMIA/SIFI [1-3]

${ }^{\mathrm{f}}$ Uses median duration of effect of 3 months

${ }^{\mathrm{g}}$ Assumption based on discussions with UK clinicians

${ }^{\mathrm{h}}$ Assumption based on discussions with UK clinicians

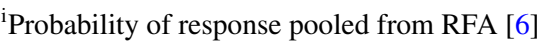

${ }^{j}$ Uses median duration of effect. Assumed 7.9 months based on evidence from RFA [7]

To fully explore the uncertainty in the model parameters, the probabilistic sensitivity analysis (PSA) varied all variable model inputs simultaneously, using Excel's random number generator to sample values from the probability distributions for each variable as detailed in Table 2 . This was repeated for 1000 iterations using a custom visual basic for applications (VBA) script following an approach outlined by Briggs et al. [35] and was sufficient to achieve stabilization.

\section{Results}

\subsection{Base-Case Results}

The results of the base-case analysis are presented in Table 4 . After 5 years in the NSM arm, patients on the stepped treatment (strategy 1) had received an average of 0.98 steroid injections, 1.30 courses of physical therapy, and 1.22 RFA procedures. Almost all patients had stopped treatment, and only $4.3 \%$ were living with improved pain and function. This contrasts with the MI SIJF arm, where $64.3 \%$ remain in the response-to-treatment health state after 5 years, with an associated improvement in pain and physical function. Surgical intervention with MI SIJF accrued total procedurerelated and pain-management costs of $£ 8358$, while the stepped treatment strategy had total costs of $£ 6880$. Over the 5-year time horizon, the model predicts that patients with MI SIJF would have 2.98 QALYs (discounted) compared to strategy 1 with 2.30 QALYs (discounted). This resulted in an incremental cost-effectiveness ratio (ICER) for MI SIJF versus strategy 1 of $£ 2164 / \mathrm{QALY}$ gained.

Strategy 2 of the NSM arm had lower costs (£6564) than strategy 1 because there were fewer procedures of all types ( 0.66 RFA, 0.49 corticosteroid injections, and 0.65 physiotherapy courses), and this resulted in 2.26 QALYs and an ICER of $£ 2468 /$ QALY gained for MI SIJF. Strategy 3 of the NSM arm had lower costs (£6580) than strategy 1 because there were no PTSI procedures and just a slight increase in RFA procedures (1.33 RFA). This resulted in 2.28 QALYs and an ICER of £2518/QALY gained for MI SIJF. MI SIJF results in greater utility gain compared to all non-surgical treatment pathways. Within the NSM arms, the stepped pathway resulted in a higher utility gain than PTSI or RFA alone because patients had the chance for additional treatment having failed the firstline intervention.

\subsection{Sensitivity Analyses}

The results of the PSA shown in the cost-effectiveness plane (CEP) (Fig. 3a) illustrate that all three treatment strategies are broadly consistent in terms of their results. The majority of simulated ICERs occupy the northeast quadrant of the CEP, where there exists a trade-off between increased cost and increases in QALYs gained. The cost-effectiveness acceptability curve (CEAC) (Fig. 3b) highlights that MI SIJF has a probability of being the dominant treatment of $5 \%, 4 \%$, and $4 \%$ versus strategies 1,2 , and 3, respectively. It is also apparent that at a threshold of $£ 20,000 / \mathrm{Q} A L Y$ gained, MI SIJF has a probability of being cost-effective of $96 \%, 97 \%$, and $91 \%$ versus non-surgical treatment for strategies 1,2 , and 3 , respectively. 
Table 4 Cost-effectiveness results in the base case

\begin{tabular}{|c|c|c|c|c|c|c|c|}
\hline & \multirow[t]{2}{*}{ MI SIJF } & \multicolumn{6}{|c|}{ NSM strategies } \\
\hline & & Strategy 1 & Diff. & Strategy 2 & Diff. & Strategy 3 & Diff. \\
\hline \multicolumn{8}{|l|}{ Treatment outcomes after 5 years } \\
\hline MI SIJF procedures per patient & 1.04 & & & & & & \\
\hline RFA procedures per patient & & 1.22 & & 0.66 & & 1.33 & \\
\hline Corticosteroid injections procedures per patient & & 0.98 & & 0.49 & & & \\
\hline Physiotherapy courses per patient & & 1.30 & & 0.65 & & & \\
\hline Proportion in response & $64.3 \%$ & $4.3 \%$ & $60.0 \%$ & $0.6 \%$ & $63.7 \%$ & $1.2 \%$ & $663.1 \%$ \\
\hline \multicolumn{8}{|l|}{ Costs after 5 years } \\
\hline MI SIJF surgery costs & $£ 6322$ & & & & & & \\
\hline PTSI costs & & $£ 978$ & & $£ 489$ & & & \\
\hline RFA costs & & $£ 1092$ & & $£ 628$ & & $£ 1255$ & \\
\hline Pain-management costs & $£ 2037$ & $£ 4811$ & & $£ 5448$ & & $£ 5325$ & \\
\hline Total & $£ 8358$ & $£ 6880$ & $£ 1478$ & $£ 6564$ & $£ 1794$ & $£ 6580$ & $£ 1778$ \\
\hline \multicolumn{8}{|l|}{ Health outcomes after 5 years } \\
\hline Life years (discounted) & 4.53 & 4.53 & 0 & 4.53 & 0 & 4.53 & 0 \\
\hline QALYs (discounted) & 2.98 & 2.30 & 0.68 & 2.26 & 0.73 & 2.28 & 0.71 \\
\hline Life years (undiscounted) & 4.96 & 4.96 & 0 & 4.96 & 0 & 4.96 & 0 \\
\hline QALYs (undiscounted) & 3.27 & 2.52 & 0.75 & 2.47 & 0.80 & 2.49 & 0.78 \\
\hline ICER after 5 years (MI SIJF vs NSM strategy) & & $£ 2164$ & & $£ 2468$ & & $£ 2518$ & \\
\hline
\end{tabular}

MI SIJF minimally invasive sacroiliac joint fusion, NSM non-surgical management, ICER incremental cost-effectiveness ratio, ODI Oswestry Disability Index, PTSI physical therapy and corticosteroid injections, $Q A L Y$ quality-adjusted life year, RFA radiofrequency ablation

The results of the DSA shown in the tornado plot (Fig. 4) illustrate that the base-case result for all strategies is most sensitive to the input determining the proportion of patients that achieve a response to treatment with MI SIJF. However, changing this value by $20 \%$ in either direction does not change the conclusion that MI SIJF is cost-effective in the medium term.

The effect of the time horizon was explored by extending the model to a patient's lifetime. The results are shown in the electronic supplementary material (Table S1). When comparing all the treatment strategies to MI SIJF, all are dominated, i.e. they are more costly and less effective than the comparator. A further threshold analysis was performed on the model time horizon. Variation of the model time horizon showed that MI SIJF becomes cost-effective at a threshold of $£ 20,000$ /QALY gained by year 2 and MI SIJF is dominant (cost-saving and has better outcomes) by year 7. An additional threshold analysis showed that the ICER increases when the duration of the effect of the PTSI and RFA is increased; the results show that the ICER is less than $£ 3000$ for a duration of effect of up to 24 months for both the PTSI and RFA interventions across all three treatment strategies.

\section{Discussion}

This analysis demonstrates that for patients with chronic SIJ pain, MI SIJF is cost-effective compared to NSM in an NHS context over a 5-year time horizon. Although the difference in cost between MI SIJF and NSM is relatively small ( $£ 8358$ vs $£ 6880$ for NSM strategy 1), there is a significant difference in QALYs as a result of the superior and durable effect of the surgical intervention (2.98 QALYs vs 2.30 QALYs for NSM strategy 1). At 5 years after MI SIJF, around 64\% of patients are expected to maintain a response in terms of reduction in ODI compared to just over $4 \%$ of patients in the NSM arm, where treatments are known to provide only temporary relief rather than a long-term pain-management solution. This resulted in an ICER for MI SIJF versus NSM strategy 1 of $£ 2164 /$ QALY gained.

As with all models, the current analysis has limitations imposed by data availability and our structural assumptions. One limitation is the difficulty in modelling the variation in NSM pathways, as there is no accepted standard practice. Feedback from consultants interviewed

prior to model development suggested regional variability in practice. Despite limited efficacy data, feedback from consultants interviewed prior to model development suggest that PTSI is a common first-line treatment, and 
Fig. 3 a Probabilistic sensitivity analysis: cost-effectiveness plane. The dotted line represents an ICER of $£ 20,000$ per QALY gained. b Cost-effectiveness acceptability curve for MI SIFJ compared to three non-surgical treatment strategies. ICER incremental cost-effectiveness ratio, $M I$ SIJF minimally invasive sacroiliac joint fusion, $Q A L Y$ quality-adjusted life years, WTP willingness to pay

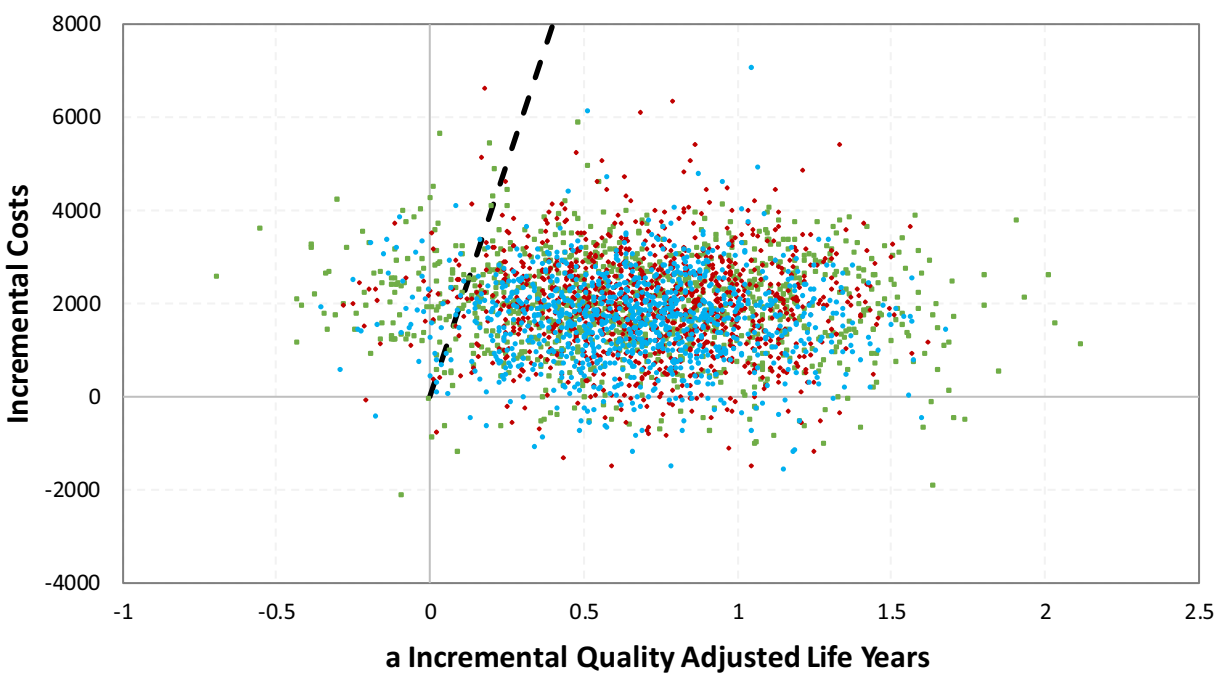

- - WTP Threshold at f20,000 per QALY - Stratgey $3 \quad$ S Strategy 2 - Strategy 1

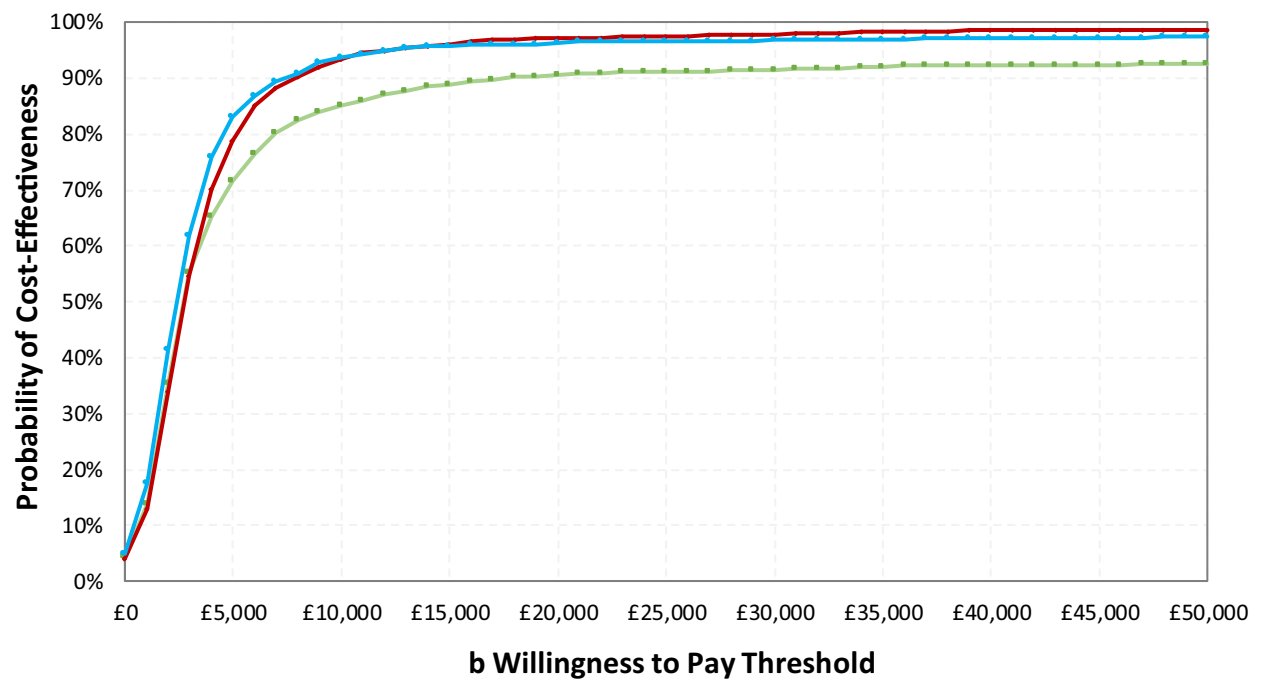

- Strategy $3 \longrightarrow$ Strategy $2 \longrightarrow$ Strategy 1

a small proportion of patients may be offered RFA at a later stage. The NICE guidelines [33] on lower back pain recommend RFA of the lumbar facets, but do not address this treatment for SIJ pain. Furthermore, these guidelines explicitly do not recommend repeat corticosteroid injections, due to a lack of robust evidence on their efficacy. Additionally, because of the lack of robust data for the efficacy of corticosteroid injections, it was necessary to group together physical therapy and corticosteroid injections as a treatment (PTSI), which might not be reflective of clinical practice in England. Despite this, by pooling the comparator arms from the two MI SIJF RCTs it meant patients who had an RFA procedure performed as part of the trial were included in the efficacy outcomes; this limitation is conservative in the sense that it should inflate the efficacy of the comparator group. The duration of the effect of both corticosteroid injections and RFA remains uncertain, but threshold analysis on these values suggested that the assumptions employed were not drivers of the results. One major model driver was the healthcare utilization cost due to pain management in non-responder health states. This cost was derived from a facet joint injection trial that failed to meet enrolment criteria, and thus the indication was not identical to the current study and the number it was based on was small; however, the cost values used were similar to those employed in the NICE MTEP analysis [22].

When considering the generalizability of the analysis, it is important to highlight that it has been conducted from the perspective of a comprehensive healthcare system, namely the NHS in England. Therefore, the resource 


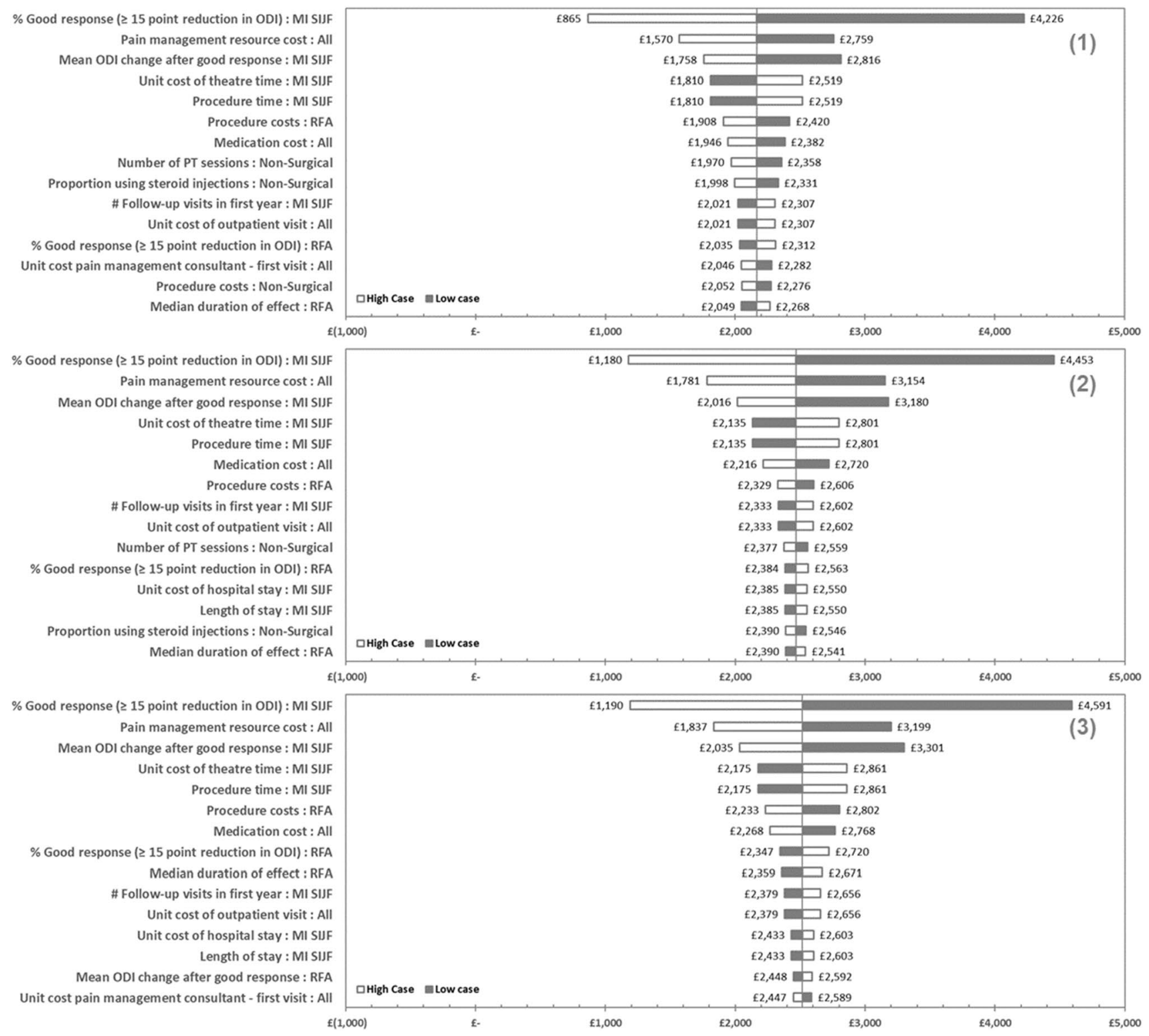

Fig. 4 Deterministic sensitivity analysis showing parameter uncertainty of $20 \%$ of the ICER of MI SIJF vs three NSM treatment strategies. ICER incremental cost-effectiveness ratio, MI SIJF minimally

utilization and cost data that have been used are not necessarily generalizable to other locations.

Further, the assumptions used in the NSM arm of the model are quite conservative, with a limited number of repeat injections or RFA procedures per patient occurring during the 5 years. This is different from NSM treatment in other geographies, where repeat procedures are more frequent [36, 37].

The iFuse TTIs, the clinical evidence base for which the current model builds, have several unique features, including a triangular cross section, porous surface allowing bone ingrowth/ongrowth, and that the devices are impacted into invasive sacroiliac joint fusion, $N S M$ non-surgical management, $O D I$ Oswestry Disability Index, $P T$ physical therapy, $R F A$ radiofrequency ablation

place rather than screwed into place. For these reasons, the safety and effectiveness of iFuse TTIs are likely not generalizable to other laterally placed devices which are circular in cross section, do not have a porous surface, and are screwed into place. Increased interest in SIJ dysfunction and fusion has generated other devices and allograft products placed dorsally. However, there is limited evidence supporting their safety and effectiveness, and these are not covered within NICE IPG 578 [14].

The results of our analysis align with the conclusions drawn by the NICE EAC team, who reported that MI SIJF resulted in savings of $£ 129$ per patient treated by year 8 [22]. 
This indicates that MI SIJF is likely to dominate NSM over the longer term, findings also backed up in our extended time horizon model (Table S1, see the electronic Supplementary material). More broadly, our results are also similar to other cost-utility analyses which demonstrated MI SIJF are costeffective compared to non-surgical treatments from US commercial payer and government perspectives [38].

The predicted ICER from our study is in the same range as results from other UK-focused analyses (Table S2, see the electronic Supplementary material) for orthopaedic surgical interventions. Due to different time horizons and modelling approaches, results cannot be directly compared. Nonetheless, our results suggest that MI SIJF represents comparative value when compared to treatments routinely offered within the NHS to treat severe pain and mobility issues, such as knee and hip replacement.

Sensitivity analysis suggests that there is a greater than 90\% probability of MI SIJF being considered cost-effective at a threshold of $£ 20,000 / \mathrm{QALY}$ compared to NSM across all treatment strategies. When extending the analysis to a lifetime horizon, MI SIJF has better outcomes and is less costly than NSM.

In conclusion, this analysis is complementary to the costminimization analysis conducted under the NICE MTEP evaluation and is intended to aid NHS decision makers in evaluating, with a patient-focused perspective, the long-term clinical and economic benefits of MI SIJF.

Acknowledgements David Julian, SI-BONE, Inc., San Jose, CA, USA, provided editorial support.

\section{Declarations}

Funding SI-BONE, Santa Clara, CA, USA was the primary funding source.

Conflict of interest Rob S. Blissett and Deirdre B. Blissett are directors of MedTech Economics Ltd., a consultancy commissioned by Si-BONE Inc. to perform economic analysis and draft this article. Daniel J. Cher and W. Carlton Reckling are employed by Si-BONE Inc. Matthew P. Newton Ede has received funding from Si-BONE Inc. to provide training unrelated to this article. Philip M. Stott has received funding to lecture on behalf of by Si-BONE Inc. unrelated to this article.

Ethics approval Not applicable.

Consent to participate Not applicable.

Consent for publication Not applicable.

Availability of data and material All data inputs used in the model are available within the article and the electronic supplementary material.

Code availability Not applicable.

Author contributions Conceived and designed the experiments: RSB, DBB, DJC, and WCR. Analysed the data: RSB, DBB, DJC, and WCR.
Contributed reagents/materials/analysis tools: RSB, DBB, DJC, and WCR. Wrote the paper: RSB, DBB, DJC, and WCR. Interpretation of the analysis: RSB, DBB, DJC, and WCR. Played a role in the design and the execution of the study and commented on the manuscript: RSB, DBB, DJC, WCR, MPNE, and PMS.

Open Access This article is licensed under a Creative Commons Attribution-NonCommercial 4.0 International License, which permits any non-commercial use, sharing, adaptation, distribution and reproduction in any medium or format, as long as you give appropriate credit to the original author(s) and the source, provide a link to the Creative Commons licence, and indicate if changes were made. The images or other third party material in this article are included in the article's Creative Commons licence, unless indicated otherwise in a credit line to the material. If material is not included in the article's Creative Commons licence and your intended use is not permitted by statutory regulation or exceeds the permitted use, you will need to obtain permission directly from the copyright holder. To view a copy of this licence, visit http://creativecommons.org/licenses/by-nc/4.0/.

\section{References}

1. Savigny P, Watson P, Underwood M. Early management of persistent non-specific low back pain: summary of NICE guidance. BMJ. 2009;338(jun04 3):b1805. https://doi.org/10.1136/bmj. b1805.

2. Cohen SP, Chen Y, Neufeld NJ. Sacroiliac joint pain: a comprehensive review of epidemiology, diagnosis and treatment. Expert Rev Neurother. 2013;13(1):99-116. https://doi.org/10.1586/ ern.12.148.

3. DePalma MJ, Ketchum JM, Saullo TR. Etiology of chronic low back pain in patients having undergone lumbar fusion. Pain Med. 2011;12(5):732-9. https://doi.org/10.111 $1 /$ j.1526-4637.2011.01098.x.

4. Liliang P-C, Lu K, Liang C-L, Tsai Y-D, Wang K-W, Chen H-J. Sacroiliac joint pain after lumbar and lumbosacral fusion: findings using dual sacroiliac joint blocks. Pain Med. 2011;12(4):565-70. https://doi.org/10.1111/j.1526-4637.2011.01087.x.

5. Whitehurst DGT, Bryan S, Lewis M, Hill J, Hay EM. Exploring the cost-utility of stratified primary care management for low back pain compared with current best practice within risk-defined subgroups. Ann Rheum Dis. 2012;71(11):1796-802. https://doi. org/10.1136/annrheumdis-2011-200731.

6. Maniadakis N, Gray A. The economic burden of back pain in the UK. Pain. 2000;84(1):95-103. https://doi.org/10.1016/S0304 -3959(99)00187-6.

7. Murray CJL, et al. Disability-adjusted life years (DALYs) for 291 diseases and injuries in 21 regions, 1990-2010: a systematic analysis for the Global Burden of Disease Study 2010. Lancet. 2012;380(9859):2197-223. https://doi.org/10.1016/S0140 $-6736(12) 61689-4$

8. Zheng P, Schneider BJ, Yang A, McCormick ZL. Image guided sacroiliac joint injections; an evidence-based review of best practices and clinical outcomes. PM\&R. 2019. https://doi. org/10.1002/pmrj.12191.

9. Kennedy DJ, Engel A, Kreiner DS, Nampiaparampil D, Duszynski B, MacVicar J. Fluoroscopically guided diagnostic and therapeutic intra-articular sacroiliac joint injections: a systematic review. Pain Med. 2015;16(8):1500-18. https://doi.org/10.1111/ pme.12833. 
10. Simopoulos TT, et al. Systematic review of the diagnostic accuracy and therapeutic effectiveness of sacroiliac joint interventions. Pain Physician. 2015;18(5):E713-756.

11. Yang AJ, McCormick ZL, Zheng PZ, Schneider BJ. Radiofrequency ablation for posterior sacroiliac joint complex pain: a narrative review. PM\&R. 2019. https://doi.org/10.1002/pmrj.12200.

12. Sun H-H, Zhuang S-Y, Hong X, Xie X-H, Zhu L, Wu X-T. The efficacy and safety of using cooled radiofrequency in treating chronic sacroiliac joint pain: a PRISMA-compliant meta-analysis. Medicine (Baltimore). 2018;97(6):e9809. https://doi.org/10.1097/ MD.0000000000009809.

13. Buchowski JM, Kebaish KM, Sinkov V, Cohen DB, Sieber AN, Kostuik JP. Functional and radiographic outcome of sacroiliac arthrodesis for the disorders of the sacroiliac joint. Spine J. 2005;5(5):520-8. https://doi.org/10.1016/j.spinee.2005.02.022 ((discussion 529)).

14. National Institute for Care and Health Excellence (NICE). Minimally invasive sacroiliac joint fusion surgery for chronic sacroiliac pain: Interventional procedures guidance IPG578. London: National Institue for Health and Care Excellence (NICE); 2017.

15. Polly DW, et al. Two-year outcomes from a randomized controlled trial of minimally invasive sacroiliac joint fusion vs. non-surgical management for sacroiliac joint dysfunction. Int J Spine Surg. 2016. https://doi.org/10.14444/3028.

16. Sturesson B, Kools D, Pflugmacher R, Gasbarrini A, Prestamburgo D, Dengler J. Six-month outcomes from a randomized controlled trial of minimally invasive SI joint fusion with triangular titanium implants vs. conservative management. Eur Spine J. 2017;26(3):708-19. https://doi.org/10.1007/s00586-016-4599-9 ((Epub 2016 May 14))

17. Smith AG, et al. Open versus minimally invasive sacroiliac joint fusion: a multi-center comparison of perioperative measures and clinical outcomes. Ann Surg Innov Res. 2013;7(1):14. https://doi. org/10.1186/1750-1164-7-14.

18. Ledonio CGT, Polly DW, Swiontkowski MF. Minimally invasive versus open sacroiliac joint fusion: are they similarly safe and effective? Clin Orthop. 2014;472(6):1831-8. https://doi. org/10.1007/s11999-014-3499-8

19. Ledonio C, Polly D, Swiontkowski MF, Cummings J. Comparative effectiveness of open versus minimally invasive sacroiliac joint fusion. Med Devices Evid Res. 2014;2014(7):187-93. https://doi. org/10.2147/MDER.S60370.

20. Whang PG, et al. Long-term prospective clinical and radiographic outcomes after minimally invasive lateral transiliac sacroiliac joint fusion using triangular titanium implants. Med Devices Evid Res. 2019;12:411-22. https://doi.org/10.2147/MDER.S219862 ((Epub 2019 Sep 26)).

21. Vanaclocha V, Herrera JM, Sáiz-Sapena N, Rivera-Paz M, Verdú-López F. Minimally invasive sacroiliac joint fusion, radiofrequency denervation, and conservative management for sacroiliac joint pain: 6-year comparative case series. Neurosurgery. 2018;82(1):48-55. https://doi.org/10.1093/neuros/nyx185.

22. Dale M, Evans J, Carter K, O'Connell S, Morgan H, CarolanRees G. iFuse implant system for treating chronic sacroiliac joint pain: a NICE medical technology guidance. Appl Health Econ Health Policy. 2019. https://doi.org/10.1007/s40258-01900539-7.

23. D. J. Evans. CF14 4UJ. 2017, p. 167. www.cedar.wales.nhs.uk. Accessed 1 April 2020

24. Cher D, Wroe K, Reckling WC, Yerby S. Postmarket surveillance of 3D-printed implants for sacroiliac joint fusion. Med Devices Auckl NZ. 2018;11:337-43. https://doi.org/10.2147/ MDER.S180958.

25. Dutta K, Dey S, Bhattacharyya P, Agarwal S, Dev P. Comparison of efficacy of lateral branch pulsed radiofrequency denervation and intraarticular depot methylprednisolone injection for sacroiliac joint pain. Pain Physician. 2018;21(5):489-96.

26. Kim WM, Lee HG, Jeong CW, Kim CM, Yoon MH. A randomized controlled trial of intra-articular prolotherapy versus steroid injection for sacroiliac joint pain. J Altern Complement Med. 2010;16(12):1285-90. https://doi.org/10.1089/ acm.2010.0031.

27. Copay AG, Cher DJ. Is the Oswestry Disability Index a valid measure of response to sacroiliac joint treatment? Qual Life Res. 2016;25(2):283-92. https://doi.org/10.1007/s11136-015-1095-3 ((Epub 2015 Aug 6)).

28. Dengler J, et al. Predictors of outcome in conservative and minimally invasive surgical management of pain originating from the sacroiliac joint: a pooled analysis. Spine. 2017;42(21):1664-73. https://doi.org/10.1097/BRS.0000000000002169 ((Epub 2017 Mar 27)).

29. Vleeming A, Albert HB, Östgaard HC, Sturesson B, Stuge B. European guidelines for the diagnosis and treatment of pelvic girdle pain. Eur Spine J. 2008;17(6):794-819. https://doi. org/10.1007/s00586-008-0602-4.

30. Patel N, Gross A, Brown L, Gekht G. A randomized, placebocontrolled study to assess the efficacy of lateral branch neurotomy for chronic sacroiliac joint pain. Pain Med. 2012;13(3):383-98. https://doi.org/10.1111/j.1526-4637.2012.01328.x.

31. van Tilburg CWJ, Schuurmans FA, Stronks DL, Groeneweg JG, Huygen FJPM. Randomized sham-controlled double-blind multicenter clinical trial to ascertain the effect of percutaneous radiofrequency treatment for sacroiliac joint pain: three-month results. Clin J Pain. 2016. https://doi.org/10.1097/AJP.000000000000035 1.

32. Juch JNS, et al. Effect of radiofrequency denervation on pain intensity among patients with chronic low back pain: the mint randomized clinical trials. JAMA. 2017;318(1):68-81. https://doi. org/10.1001/jama.2017.7918.

33. NICE. Low back pain and sciatica in over 16s: assessment and management: NICE Guidelin [NG59]. NICE guideline [NG59]. 2016. https://www.nice.org.uk/guidance/ng59/chapter/recom mendations\#non-invasive-treatments-for-low-back-pain-and-sciat ica. Accessed 03 Apr 2018.

34. Fleurence RL, Hollenbeak CS. Rates and probabilities in economic modelling: transformation, translation and appropriate application. PharmacoEconomics. 2007;25(1):3-6. https://doi. org/10.2165/00019053-200725010-00002.

35. Briggs AH, Claxton K, Sculpher MJ. Decision modelling for health economic evaluation. Oxford: Oxford University Press; 2006.

36. Hawkins J, Schofferman J. Serial therapeutic sacroiliac joint injections: a practice audit. Pain Med. 2009;10(5):850-3. https://doi. org/10.1111/j.1526-4637.2009.00651.x.

37. Manchikanti L, Pampati V, Falco FJE, Hirsch JA. Growth of spinal interventional pain management techniques: analysis of utilization trends and Medicare expenditures 2000 to 2008. Spine. 2013;38(2):157-68. https://doi.org/10.1097/BRS.0b013e3182 $67 f 463$.

38. Cher DJ, Frasco MA, Arnold RJ, Polly DW. Cost-effectiveness of minimally invasive sacroiliac joint fusion. Clin Outcomes Res. 2016;8:1-14. https://doi.org/10.2147/CEOR.S94266.

39. Cohen SP, Hurley RW, Buckenmaier CC, Kurihara C, Morlando B, Dragovich A. Randomized placebo-controlled study evaluating lateral branch radiofrequency denervation for sacroiliac joint pain. Anesthesiology. 2008;109(2):279-88. https://doi.org/10.1097/ ALN.0b013e31817f4c7c.

40. Cher DJ, Reckling WC, Capobianco RA. Implant survivorship analysis after minimally invasive sacroiliac joint fusion using the iFuse implant system. Med Devices Evid Res. 2015;8:485-92. https://doi.org/10.2147/MDER.S94885. 
41. Fletcher D, Edwards D, Tolchard S, Baker R, Berstock J. Improving theatre turnaround time. BMJ Qual Improv Rep. 2017;6(1):u219831.u8131. https://doi.org/10.1136/bmjquality .u219831.w8131.

42. Heiney J, Capobianco R, Cher D. A systematic review of minimally invasive sacroiliac joint fusion utilizing a lateral transarticular technique. Int J Spine Surg. 2015. https://doi.org/10.14444 12040 .

43. Curtis L, Burns A. Unit costs of health and social care. London: PSSRU; 2016.
44. Whang PG, et al. Sacroiliac joint fusion using triangular titanium implants vs. non-surgical management: six-month outcomes from a prospective randomized controlled trial. Int J Spine Surg. 2015. https://doi.org/10.14444/2006.

45. Ellard DR, et al. Facet joint injections for people with persistent non-specific low back pain (Facet Injection Study): a feasibility study for a randomised controlled trial. Health Technol Assess. 2017;21(30):1-184. https://doi.org/10.3310/hta21300. 